\title{
FAST REPETITION RATE (FRR) FLUOROMETER FOR MAKING in situ MEASUREMENTS OF PRIMARY PRODUCTIVITY
}

\author{
Zbigniew S. Kolber and Paul G. Falkowski \\ Oceanographic and Atmospheric Science Division, Brookhaven National Laboratory, BNL- 47921 \\ Upton, New York 11973 \\ DE92 041318
}

\section{ABSTRACT}

Understanding the ocean carbon cycle and predicting how climate-induced changes in ocean circulation will affect ocean productivity requires that (a) primary productivit; be meazured with high spatial and temporal resolution, and (b) natural variability in primary productivity be parameterized with regar'd to environmental factors such as nutrient availability, irradiance, and temperature. Instrumentation to measure primary productivity from the stimulated in vivo nuorescence of phytoplankton chlorophyll is currently being developed at Brookhaven National Laboratory. The instrumentation is based on fast repetition rate (FRR) nuorometry, and provides a robust technique for deriving the photosynthetic rates in silu. Moreover, the FRR methodology directly measures severai photosynthetic parameters such as effective absorption cross section, photoconversion efficiency, and turnover lime of photosynthesis, and relates them to primary productivity. Since photosynthetic parameters are affected by environmental factors such as light and nutrient availability, the relationship between these parameters and primary productivity can be established. By understanding such relationships, prognostic models of primary productivity can be developed and parameterized.

\section{INTRODUCTION}

Primary productivity, as the first step in a series of carbon transformations, determines the scale and dynamics of carbon cycle in the ocean. Primary productivity is controlled by environmental parameters such as solar irradiance, autrient availability, trace metal concentration, and temperature. All of these parameters are highly variable both spatially and temporarily. Estimates of the primary productivity based solely on phytoplankton biomass and irradiance are unsatisfactory, and ${ }^{14} \mathrm{C}$-based measurements, are difficult to interpret.
As an alternative approach, fluorescence methods of assessing primary productivity are based on a functional relationship between the in vivo fluorescence signal of marine phytoplankton and their photosynthetic efficiency. Two basic methods have evolved during the last fow years: passive fluorescence sensors, utilizing a solar-stimulated fluorescence signal [1], and pump-and-probe fluorometry, using flashstimulated fluorescence $[2,3]$. The passive fluorescence method is based on empirical relationship between the yield of fluorescence and photosynthesis. Active fluorescence, on the other hand, is based on measurements of photosynthetic parameters such as the effective absorption cross-section. photoconversion efficiency, and turnover time of photosynthesis [4]. These parameters can be incorporated into a mechanistic model of photochemistry, based on the kinetics of electron flow between Photosystems II and I.

Fast Repetition Rate (FRR) fluorometry is based on the same fluorescence/photosynthesis relationship as pump-andprobe fluorometry [4], but utilizes a different approach to measure the photosynthetic parameters. Specifically, instead of the probe-pump-probe sequences of flashes [4], it uses a series of 30.60 flashes at $100-200 \mathrm{kHz}$ repetition rate to stimulate Photosystem II. This approach significantly shortens the experimental protocol, allows more robust measurements of photosynthetic parameters, and significantly increases the signal to noise ratio for the in situ measurements of primary productivity at low chlorophyll concentration.

\section{BACKGROUND}

We assume a model of Photosystem II as shown in Fig. 1 , where LHCII is the light absorbing anterna containing reaction center $R C I I, Q_{A}$ is the first stable electron acceptor

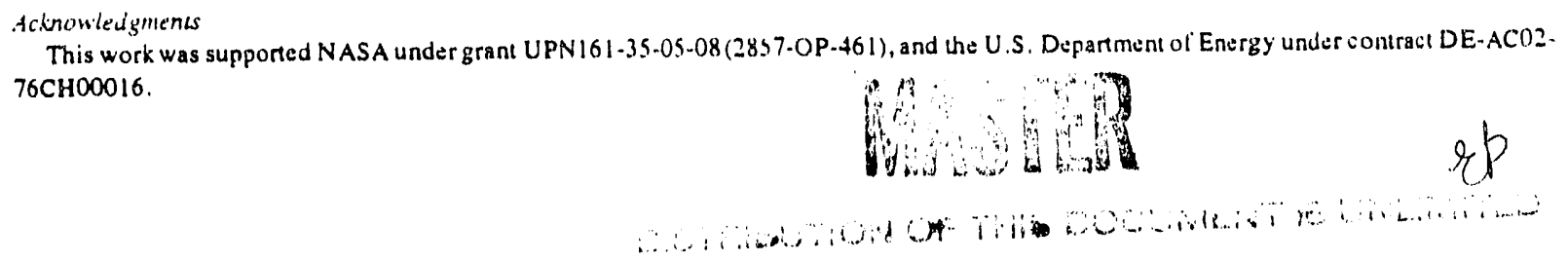


of Photosystem II, and $\mathrm{PQ}$ is the Plastoquinone pool. P680, $\mathrm{Z}$, and SO-S3 are the component of the donor side of PSII. The photosynthetic rate, $P$, can be expressed as a product of irradiance (I), the effective absorption cross section of Photosystem II $\left(\sigma_{\text {psII }}\right)$, the yield of charge separation $\left(\Phi_{q}\right)$, the yield of election transport from PSII to ?SI $(\Phi)$, and photoconversion efficiency, $\Delta \phi_{\text {aal }}$,

$$
P=I * \sigma_{p e l 1} * \Phi_{q} * \Phi_{1} * \Delta \phi_{\text {sel }}
$$

The effective absorption cross section describes the efficiency of light utilization by phytoplankton, and is determined by the effective size of the antenna and the efficiency of excitation transfer to Reaction Center II. $\Phi_{q}$ and $\Phi_{1}$ are controlled by the rate of excitation delivery to RCII $\left(=I^{*} \sigma_{\text {psII }}\right)$, and the rate

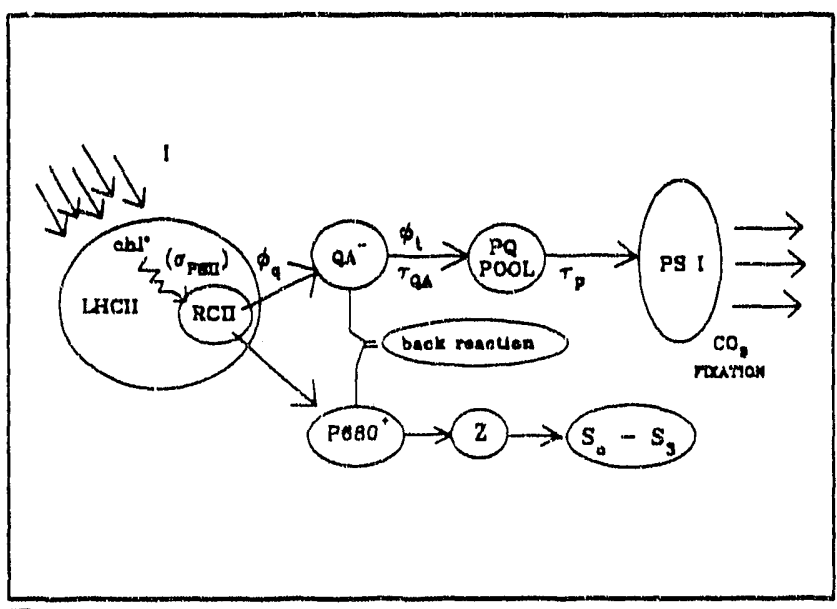

Figure 1. Model of Photosystem II.

of electron transport from PSII to PSI. $\Delta \phi_{\text {at }}$ is determined by the proportion of photosynthetically active reaction centers in phytoplankton population.

Parameters $\sigma_{\mathrm{PSI}}$ and $\Delta \phi_{\text {at }}$ strongiy depend on environmental conditions such as growth irradiance and nutrient availability [5], whereas $\Phi_{\mathrm{q}}$ and $\Phi_{\mathrm{t}}$ are determined by the time constants of oxidation of $Q_{A}\left(\tau_{Q N}\right)$, and PQ $\left(\tau_{p}\right)$. Derivation of primary production requires that these parameters be measured in situ in real time. Knowiedge of photosynthetic parameters can also provide an understanding of phytoplankton physiology.

\section{FAST REPETITION RATE (FRR) FLUOROMETER}

Fast Repetition Rate (FRR) fluorometer uses a single excitation channel to provide a series of thashes with controlled intensity and repetition rate. The subsequent flashes close more and more PSII reaction centers, resulting in a gradual increase of the fluorescence yield of phytoplankton chlorophyll. Formally, the observed fluorescence signal is a convolution of the excitation signal and the kinetics of variable fluorescence decay, and can be expressed as follow:

$$
\begin{aligned}
F_{n}=F_{o} & +\left(F_{1}-F_{2}\right) *\left[1-\exp \left(-E_{n}^{*} \sigma_{P S I I}\right)\right] * \\
& *\left\{\sum_{j=0}^{n-1} \exp \left[-j *\left(E_{n}^{*} \sigma_{P S I I}+\Delta t / \tau_{d \infty c}\right)\right]\right\},
\end{aligned}
$$

where $F_{0}$ is the fluorescence yield in the dark, $F_{0}$ is the fluorescence yield when all PSII reaction centers are closed, $E_{n}$ is the intensity of the $n$-th flash, $\Delta t$ is the time delay between flashes, and $\tau_{\text {doc }}$ is the time constant of the variable fluorescence decay.

The FRR fluorometer can operate in two different modes: a pumping mode, and a relaxation mode. In the pumping mode, excitation flashes are applied at a 100-200 $\mathrm{kHz}$ repetition rate at about $10 \%$ saturation intensity. Since the time interval between flashes $(5-10 \mu \mathrm{s})$ is much to short to allow $Q_{A}$ to become reoxidized, there will be a cumulative increase in the level of reduced $Q_{\beta}$, resulting in a gradual saturation of the fluorescence signal. The $F_{0}, F_{n}$, and $\sigma_{p s i}$ can be estimated by fitting Eqn. 2 to the measured fluorescence signal $F_{i}$. Then, the photoconversion efficiency, $\Delta \phi_{\mathrm{s}, 1}$, and the yield of charge separation, $\Phi_{q}$ is calculated as:

$$
\begin{aligned}
& \Delta \phi_{\text {nal }}=\left(F_{0}-F_{0}\right) / F_{s}, \\
& \Phi_{q}=\left(F_{0}-F_{2}\right) /\left(F_{a}-F_{0}\right),
\end{aligned}
$$

where $F_{4}$ is the same signal as $F_{0}$, but measured under ambient irradiance.

In the relaxation mode, the flash repetition rate is reduced to about $10 \mathrm{kHz}$, allowing the fluorescence yield to decay to its initial level. The kinetics of fluorescence decay depend on the amount of excitation energy transferred to PSII during the pumping sequence, and on ambient irradiance. If a pumping sequence is applied in the dark, and discontinued after saturating $Q_{\wedge}(P Q$ pool is still oxidized), the fluorescence yield decays with time constant of $Q_{A}$ oxidation, $\tau_{Q A} \sim 200-600 \mu \mathrm{s}$. Ii the relaxation sequence is applied under conditions where the PQ pool is reduced, either as a result of high ambient irradiance or prolonged pumping sequence, then fluorescence yield will decay with a time 
constant of PQ pool oxidation, which corresponds to the turnover time of Photosystem II, $\tau_{p} \sim 3-10 \mathrm{~ms}$.

The block diagram of FRR fluorometer is presented in Fig. 2. The controller, Tattletale 7 from Onset Computer, based on M68300 microprocessor family, controls the excitation sequence, data acquisition process, and performs initial data reduction. The flash control unit provides pulses of current of specified length (0.6-2.5 $\mu \mathrm{s})$ and repetition rate $(5-200 \mathrm{kHz})$ to the xenon flashlamp. The light from the flashlamp is collected, filtered, and collimated on a $1 \mathrm{~cm}^{3}$ the reference signal:

$$
F_{n}=\sum_{i=1}^{k}\left(f_{i}^{*} x_{i}\right) / \sum_{i=1}^{k}\left(x_{i}^{*} x_{i}\right)
$$

where $F_{a}$ is the fluorescence yield at $n$-th flash, $f_{i}$ and $x_{1}$ are the sampled emission and reference signal at $a$-th flash.

Examples of emission/excitation traces over a $200 \mu \mathrm{s}$ pumping sequence are presented in Fig. 3.

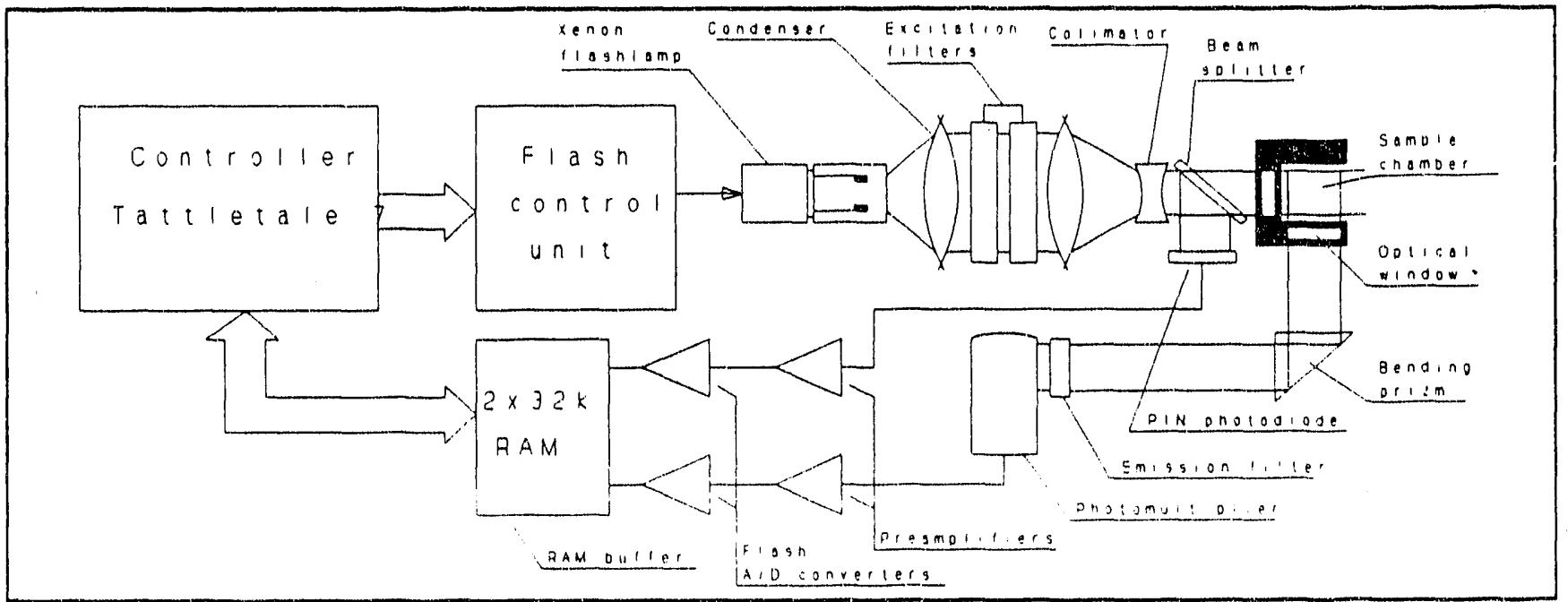

Figure 2. Block diagram of FRR fluorometer.

sample volume. A fraction of the excitation light $(\sim 2 \%)$ is collected on a PIN photodiode to provide a reference signal. The excitation light is filtered with a combination of color glass filters and low-pass interference filter to select the excitation bandwidth in a range $400-520 \mathrm{~nm}$. The emission light is collected at $90^{\circ}$ angle, bent at $90^{\circ}$ by a prism, filtered by a set of two interference filters $(685 \mathrm{~nm}, 10 \mathrm{~nm}$ bandwidth) and detected by a side-on photomulciplier tube. Both the reference and emission signals are converted at 10 $\mathrm{MHz}$ rate by 8 bit flash D/A converters, and the information is stored in a scratch-pad RAM. After completion of the flashing sequence the digitized emission and reference signals are transferred to the controller for initial data reduction. The fluorescence yield is calculated either as a ratio of emission and reference signal integrated over the length of the flash,

$$
F_{n}=\sum_{i=1}^{k} f_{i} / \sum_{i=1}^{k} x_{i}
$$

or as the product of least square fit of the emission signal to
Note that the fluorescence yield increases not only between the flashes, but during the flash as well, demonstrating that Eqn. 1. is merely a discrete approximation of the fluorescence signal changes during pumping sequence.

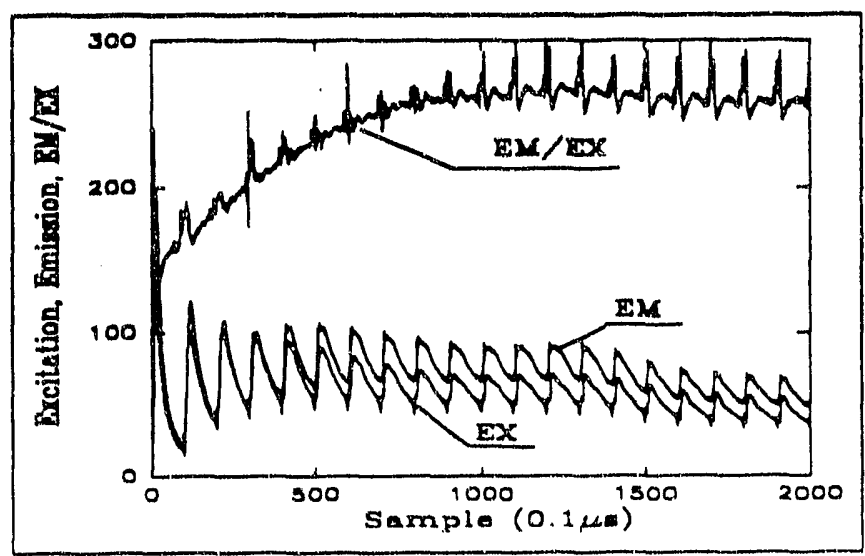

Fïgure 3. Exwilation and emission traces of FRR liucrometir. 


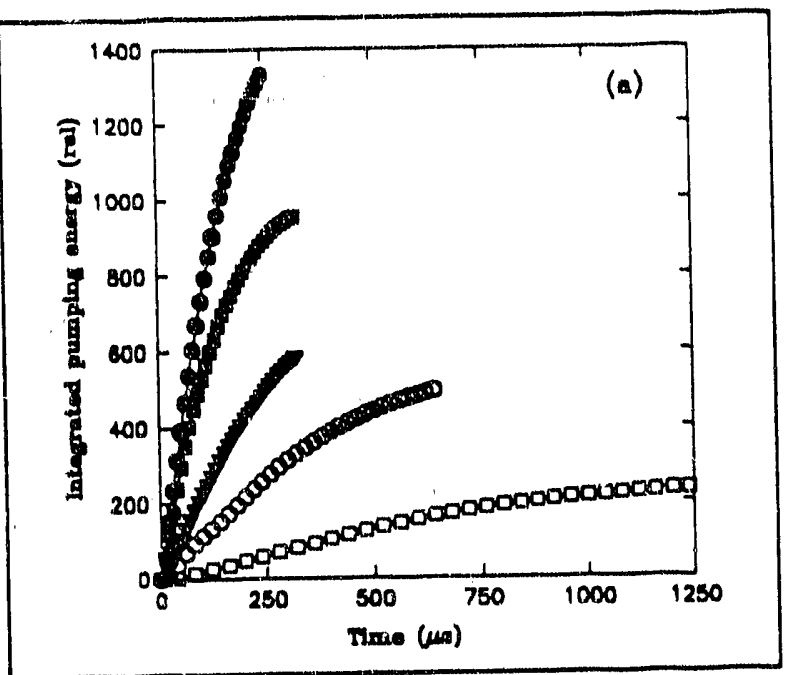

Figure, 4a. Integrated intensity of the FRR excitation signa! for varying excitation energy.

FRR operation for varying intensities of the excitation signal is demonstrated in Fig 4a, b.

The highest intensity excitation signal corresponds to the pumping mode of FRR operation, where the fluorescence signal saturates within $60-120 \mu \mathrm{s}$. At low excitation intensities, fluorescence does not saturate, and the transient increase in the fluorescence yield is followed by a relaxation of the fluorescence signal with the kinetics corresponding to the rate of electron flow between Photosystem II and Photosystem I.

\section{FIELD TESTS OF FRR FLUOROMETER}

A bench model FRR fluorometer was first used in the field in March 1992 aboard R/V Wecoma (FeLine II Cruise). . An example data set acquired cluring the transect from $\infty^{\circ} \mathrm{N}$, $142^{\circ} \mathrm{W}$ to $23^{\circ} \mathrm{N}, 142^{\circ} \mathrm{W}$ is presented in Fig. 5.

The major goal of the cruise was to test the iron limitation hypothesis in the Equatorial Pacific [6]. Several iron and nutrient enrichment experiments were conducted onboard, however, there were no ${ }^{14} \mathrm{C}$ measurements. Note the characteristic features in distribution of $\Delta \phi_{\text {sel }}$ and $\sigma_{P S u}$ in the vicinity of the equatorial undercurrent.

$\Delta \phi_{\text {m }}$ (Fig. 5.a) is generally low at the Equator, and increases northward. Low $\Delta \phi_{\text {sal }}$ implies that the growth rates of phytoplankton are limited. Apparently, the highest values of $\sigma_{\mathrm{PSII}}\left(>320\left(\mathrm{~A}^{\circ}\right)^{2}\right)$ (Fig 5.b) were observed at two equatorial stations, and $\sigma_{\mathrm{PSI}}$ generally decreased northward of the equator. The quasi-uniform chlorophyll distribution near the equator, with shallow chlorophyll maximum at stations 37 and 38 is replaced by a deep chlorophyll

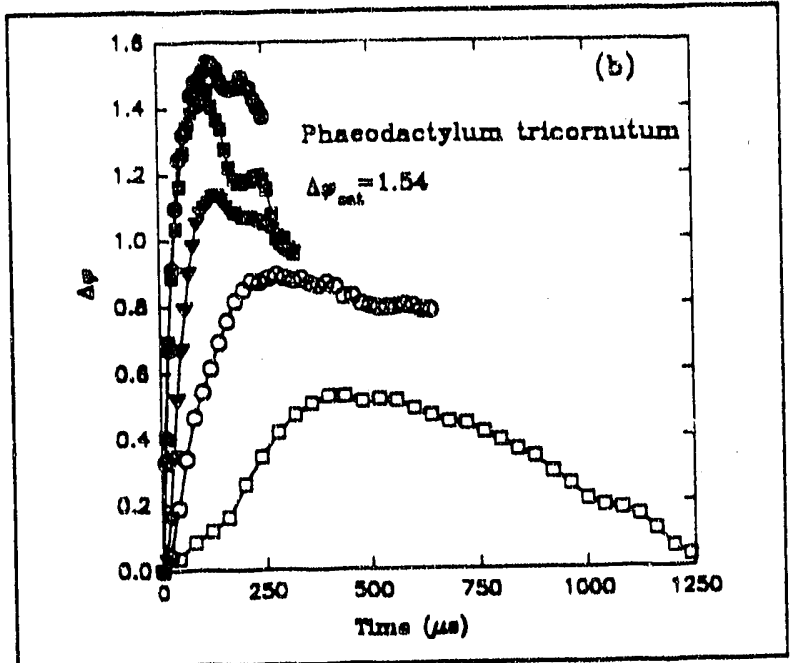

Figure $4 \mathrm{~b}$. Fluorescence response for varying intensity of the FRR excitation signal. Corresponding excitation energy as in Fig. 4 a.

maximum north of $10^{\circ} \mathrm{N}$ (Fig 5.c). The shallow chiorophyll maximum and abundance of nutrients in equatorial vaters (data not shown) further suggests that phytoplankton growth is inhibited. The possible factors inhibiting phytoplankton growth are iron limitation, excess of UV radiation, or high convective mixing rates preventing phytoplankton from adapting to high irradiances.

Both the low $\Delta \phi_{\text {sat }}$ and large $\sigma_{p s i n}$ are characteristic of from iron limitation in laboratory phytoplankton cultures [7]. Enrichment of natural phytoplankton communities with nanomolar concentration of $\mathrm{FeCl}_{3}$ lead to marked increase in $\Delta \phi_{\text {uat }}$ and chlorophyll biomass within 48 hours (data not shown), suggesting that iron limits primary production and growth rites in equatorial Pacific. We found that the nearsurface depression in $\Delta \phi_{\text {Lal }}$ is a common feature in the open ocean, and is a result of photo-oxidative damage. Using FRR methodolog, we have been abie to follow the onset of photoinhibition shortly after the sunrise, and the recovery during the night. In collaboration with $\mathrm{A}$. Hansen from the University of Rhode Island, we have established that natural levels of UV increases the photoinhibition phenomenon in the upper octiain.

The near-surface accumulation of chlorophyll biomnss a: stations 37 and 38 may be caused by the Intertropical Convergence Zone at $4^{\circ} \mathrm{N}$, which traps the phytoplankton coming from deep water at Equator. The high $\sigma_{\text {psa }}(>400$ $\left.\left(A^{\prime \prime}\right)^{2}\right)$ at $75-100$ meters at station 37 , and fast upwelling rates make the phytoplankton particularly susceptible of photoinhibition. This may further limit the primary preduction and growth rates at stations 37 and 38 , as derionstrated by low $1 \phi_{\text {sal }}$ and excess of nutrients near the surfile. 
Figure 5. Distribution of photosynthetie parameters ( $\triangle \phi$ sat, opSII), Chlorophyll, and Temperature atong a transect from $0^{\circ} \mathrm{N}, 142^{\circ} \mathrm{W}$ to $23^{\circ} \mathrm{N}, 142^{\circ} \mathrm{W}, \mathrm{as}$ measured by FRR nuorometer.

\section{CONCLUSION.}

FRR methodology allows for rapid, non-destructive, real-time estimation of phytoplankton biomass, photosynthetic rates, and the photosynthetic parameters. This methodology is adaptable to contintious mapping, flow cytoretry, and moored instnumentation. Detailed spatial and tempural maps of photosynthetic parameters obtained by FRR methodology can greatly increase our understanding of how phytoplankton production is affected by the physical and chemical structure of the upper ocean.

\section{REFERENCES}

[1] W.S, Chamberlin, C. R. Booth, D. A. Kiefer, J. H. Morrow and R. C. Murphy. "Evidence for a simple relationship between natural fluorescence, photosynthesis ad chlorophyll in the sea ", Deep-Sea Res. 37(6), pp. 951-973, 1990.

[2] P.G. Falkowski, and Z.S. Kolber, "Phytoplankton photosynthesis in the Atlantic Ocean as measured from a submersible pump and probe fluorometer in situ". Current Research in Photonnthesis IV. M Baltschewsky (ed.), Kluwer, London, pp. 923-926, 1990.

[3] P.G. Falkowski, D. Ziemman, Z.S. Kolber, and P. K. Bienfang, "Nutrient pumping and phytoplankton response in a subtropical mesoscale eddy". Nature 352, pp. $55-58,1991$.

[4] Z.S. Kolber, J. Zehr, and P.G. Falkowski, "Effects of growth irradiance and nitogen limitation on photosynthetic enurgy conversion in Photosystem II",

Plant Physiol. 88, pp. 923-929, 1998.

[5] Z.S. Kolber, and P.G. Faltowski, "Natural variability in photosynthetic energy conversion efficiency: A field study in a Gulf of Maine", Limnol. Oceanogr., 35(1), $72-79,1990$.

[6] J.H. Martin, and S.E. Fitzwater, "Iron deficiency hois phytoplankton growth in the north-east Pacific subarctic", Nature 331, 341-343, 1988.

[7] R.M. Greene, R.J.Geider, and P.G Falkowski, "Efect of iron limitation on photosynthesis in marine phytoplankton". Limmol. Oceimegr., 36(8), pp. 177\%. 1782. 1991 . 

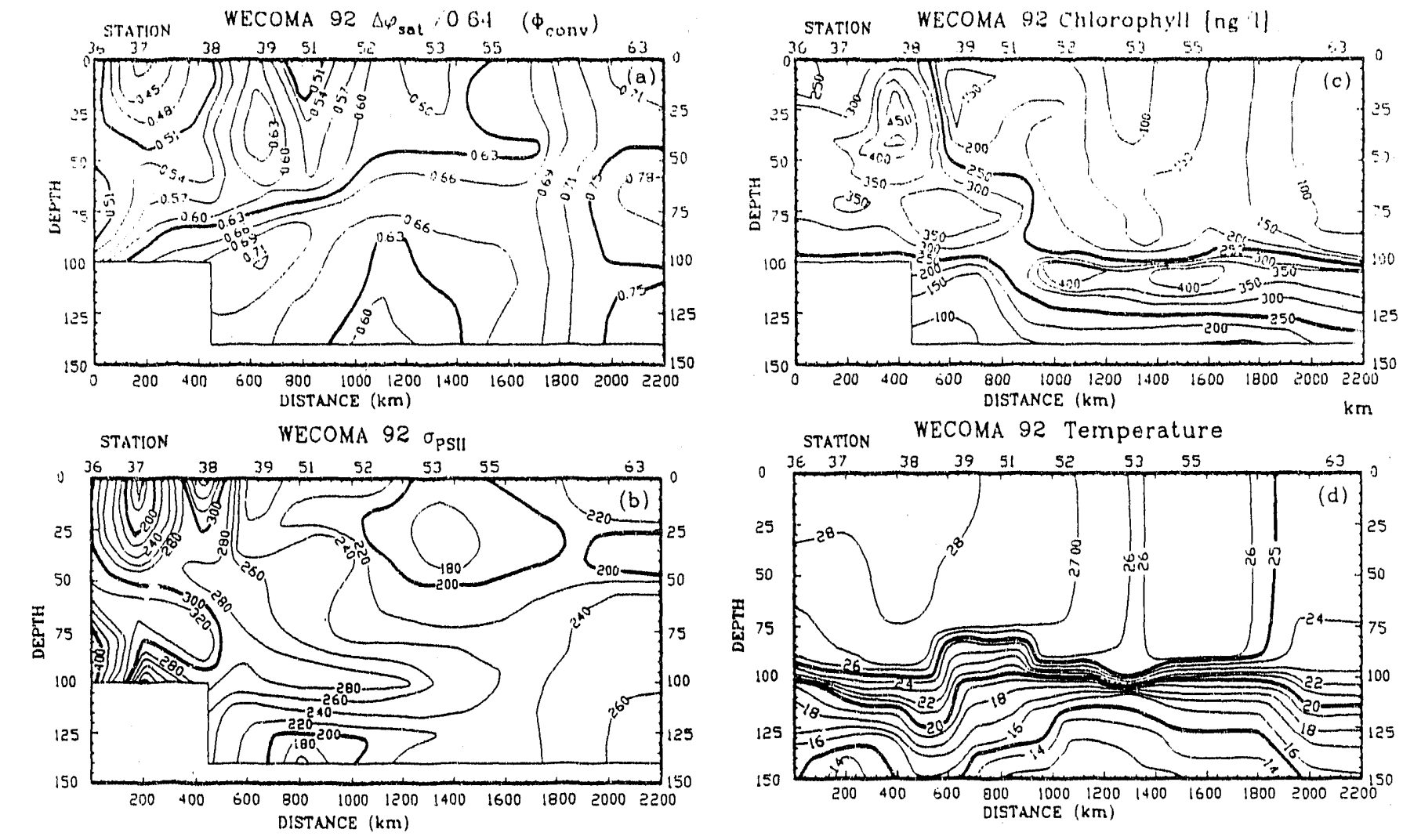


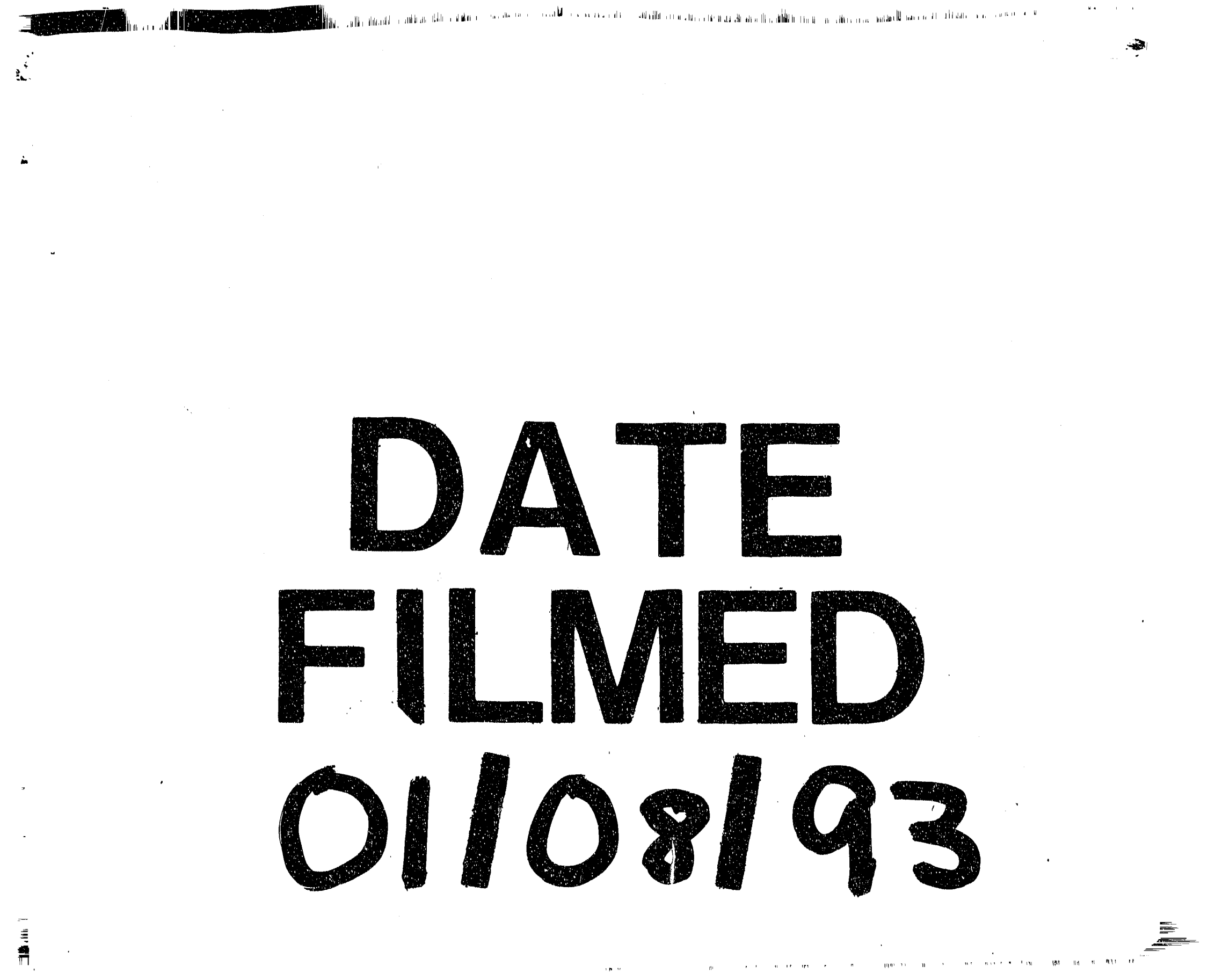


